\title{
Long-distance color-dependent quark potentials in the Coulomb gauge QCD
}

\author{
Yoshiyuki Nakagawa $^{a}$, Atsushi Nakamura $^{b}$, Takuya Saito $^{*, c}$, Toki Hiroshi ${ }^{a}$ \\ ${ }^{a}$ Research Center for Nuclear Physics, Osaka University, Ibaraki, Osaka 567-0047, Japan \\ ${ }^{b}$ Research Institute for Information Science and Education, Hiroshima University, \\ Higashi-Hiroshima 739-8521, Japan \\ ${ }^{c}$ Integrated Information Center, Kochi University, Kochi, 780-8520, Japan \\ E-mail: tsaitou@kochi-u.ac.jp
}

\begin{abstract}
We study the long-distance color-dependent potentials between two quarks in the quenched lattice QCD simulation with the Coulomb gauge fixing. This formulation enables us to define the colorCoulomb instantaneous quark potential. Numerical simulations show that the color-singlet and color-antitriplet instantaneous potentials yield attractive confining forces at large distances. We also address the divergence problem of the color-non-singlet channels.
\end{abstract}

The XXV International Symposium on Lattice Field Theory

July 30-4 August 2007

Regensburg, Germany

\footnotetext{
* Speaker.
} 


\section{Introduction}

The long-distance color-dependent forces among quarks and gluons are the key quantities for the understanding of the internal structure of hadron as well as color confinement dynamics. Quark interactions at short distances are characterized by one-gluon exchange potential, while the confinement phenomena emerging at the hadronic scale $(1 \mathrm{fm})$ can not be described in the perturbation theory.

In order to investigate the color-dependent heavy-quark potential in a lattice QCD simulation, we adopt the Coulomb gauge that has an intuitive physical picture of quark-gluon dynamics. The gluon has two physical transverse modes and the Coulomb gauge has no negative metric compared with the covariant-type gauge. In particular, the time-time-gluon component generates a colorCoulomb instantaneous interaction, which becomes important for studying quark-bound system.

The color confinement scenario in the Coulomb gauge has been extensively studied in various situations[1, 2, 3, 4, 5, 6, 7, 8, 9]. This scenario was originally discussed by Gribov [10], and in recent years, has been advocated by Zwanziger [1]. The color-Coulomb instantaneous potential produces color confinement and lattice numerical simulations have already shown that $V_{\text {coul }}(R)$ behaves as a linearly rising potential at long distances $[4,5,6,7]$.

A non-perturbative calculation on the confining color-dependent force was carried out by using the lattice QCD simulation in Ref. [11]. Using Polyakov line correlators (PLC) [12], one can calculate the color-dependent $q \bar{q}$ and $q q$ (diquark) potentials on a lattice. However, there exists the color-singlet state in nature and the singularity of the color-non-singlet channel may emerge as the lattice volume effect, which has not been investigated in the previous study [11]. Additionally, there have been some of lattice studies which mainly focused on the diquark structure $[13,14,15,16]$. It has been furthermore confirmed that in the deconfinement phase, the color-dependent potentials are color-screened [17, 18, 19, 20].

In this paper, we report lattice QCD calculations of the color-dependent two-quark potentials with the PLC in the Coulomb gauge, and mainly discuss the finite size volume effect of those color non-singlet potentials. In section 2, we summarize the importance of the color-Coulomb instantaneous potential in the Coulomb gauge. In section 3, the numerical results and the analyzes are given. Section 4 is devoted to our summary.

\section{Color-dependent potentials}

\subsection{Instantaneous potential in the Coulomb gauge QCD}

The Coulomb gauge QCD has been quantized through the Faddeev-Popov technique [1] and renormalizability of this theory has been also proved in terms of the Hamiltonian and Lagrangian formalism [2,3]. The use of the Coulomb gauge as a physical gauge leads us to classify transverse gluon modes and an instantaneous interaction, which is required to make quark bound states in analogy with QED.

The Hamiltonian of QCD in the Coulomb gauge can be given by

$$
H=\frac{1}{2} \int d^{3} x\left(E_{i}^{t r 2}(\vec{x})+B_{i}^{2}(\vec{x})\right)+\frac{1}{2} \int d^{3} x d^{3} y(\rho(\vec{x}) \mathscr{V}(\vec{x}, \vec{y}) \rho(\vec{y})),
$$


where $E_{i}^{t r}, B_{i}$ and $\rho$ are the transverse electric field, the transverse magnetic field and the color charge density, respectively. The function $\mathscr{V}$ in the second term is made by the Faddeev-Popov (FP) operator in the spatial direction, $M=-\vec{D} \vec{\partial}=-\left(\vec{\partial}^{2}+g \vec{A} \times \vec{\partial}\right)$,

$$
\mathscr{V}(\vec{x}, \vec{y})=\int d^{3} z\left[\frac{1}{M(\vec{x}, \vec{z})}\left(-\vec{\partial}_{(\vec{z})}^{2}\right) \frac{1}{M(\vec{z}, \vec{y})}\right] .
$$

From the partition function with Hamiltonian Eq. (2.1), one can evaluate the time-time gluon propagator composed of the following two parts:

$$
g^{2}\left\langle A_{0}(x) A_{0}(y)\right\rangle=g^{2} D_{00}(x-y)=V(x-y)+P(x-y),
$$

where

$$
V(x-y)=g^{2}\langle\mathscr{V}(\vec{x}, \vec{y})\rangle \delta\left(x_{4}-y_{4}\right) .
$$

The equation (2.4) is the instantaneous color-Coulomb potential at equal time and causes antiscreening, so that this potential should be a confining potential to attract quarks in hadron. Note that Eq. (2.4) in the case of QED as a non-confining theory is identified as a Coulomb propagator $\left\langle-1 / \partial_{i}^{2}\right\rangle$ or a Coulomb potential $1 / r$.

\subsection{Color-Coulomb instantaneous potential on the lattice}

In this study we employ a partial-length Polyakov line (PPL) which can be defined as $[4,5]$

$$
L(\vec{x}, T)=\prod_{t=1}^{T} U_{0}(\vec{x}, t), \quad T=1,2, \cdots, N_{t}
$$

Here $U_{0}(\vec{x}, t)=\exp \left(\operatorname{iag} A_{0}(\vec{x}, t)\right)$ is an $S U(3)$ link variable in the temporal direction and $a, g, A_{0}(\vec{x}, t)$ and $N_{t}$ represent the lattice cutoff, the gauge coupling, the time component of a gauge potential and the temporal-lattice size. A PPL correlator in the color-singlet channel is defined by

$$
G_{1}(R, T)=\frac{1}{3}\left\langle\operatorname{Tr}\left[L(R, T) L^{\dagger}(0, T)\right]\right\rangle,
$$

where $R$ stands for $|\vec{x}|$. From Eq. (2.6) one evaluates a color-singlet potential,

$$
V(R, T)=\log \left[\frac{G_{1}(R, T)}{G_{1}(R, T+a)}\right] .
$$

For the smallest temporal lattice extension we define

$$
V(R, 0)=-\log \left[G_{1}(R, 1)\right]
$$

Here $V(R, 0)$ in the Coulomb gauge is assumed to be the color-Coulomb instantaneous potential $V_{\text {coul }}(R)$. The $V(R, T)$ in the limit $T \rightarrow \infty$ becomes the Polyakov line potential. These two potentials are expected to satisfy Zwanziger's inequality, $V_{\text {phys }}(R) \leq V_{\text {coul }}(R)[21]$, where $V_{\text {phys }}(R)$ is the physical potential extracted from the Wilson loop. We finally apply the above discussion to the other $S U$ (3) color-dependent potentials between two quarks [12]. 


\subsection{Infrared divergence of the color-dependent potential}

In the Coulomb gauge QCD the color-singlet interaction only cancels the infrared divergence [5]. For the instantaneous potential with $R=x_{0}-y_{0}$ (Eq. (2.2)) we introduce the ansatz

$$
V(\vec{R})=T_{1}^{a} T_{2}^{b} \int \frac{d \vec{p}}{(2 \pi)^{3}} \frac{d^{2}(p) f(p)}{p^{2}} e^{i \vec{p} \cdot \vec{R}}
$$

where $d(p)$ is the expectation value of the Faddeev-Popov operator and $f(p)$ means the deviations of the factorization $[23,24]$. On the other hand, the self-energy interaction not depending on the distance $R$ is written as

$$
\Sigma=\left(T_{i}^{a}\right)^{2} \int \frac{d \vec{p}}{(2 \pi)^{3}} \frac{d^{2}(p) f(p)}{p^{2}}
$$

where the Casimir invariant $\left(T_{i}^{a}\right)^{2}=4 / 3$ in the fundamental representation of the $S U(3)$ group.

The infrared (not ultraviolet) divergence emerges in both Eps. (2.9) and (2.10). If the term $d^{2}(p) f(p)$ behaves as $(1 / \sqrt{p})^{2} \cdot 1 / p$ that would be responsible for the linear potential $V \sim 1 / p^{4}$, as have been analyzed in the Refs. [24, 27], then the infrared singularities unrelated to the linear potential arise from two terms:

$$
V^{I S}(\vec{R})=4 \pi\left(T_{1}^{a} T_{2}^{b}\right) \int_{0}^{\infty} d p \frac{1}{p^{2}}, \quad \Sigma^{I S}=4 \pi\left(T_{i}^{a}\right)^{2} \int_{0}^{\infty} d p \frac{1}{p^{2}} .
$$

As a result, they are completely cancelled in the case of the color-singlet representation since $\left(T_{1}^{a} T_{2}^{b}\right)+\left(T_{i}^{a}\right)^{2}=(-4 / 3)+4 / 3=0$. Meanwhile, the other cases are proportional to the following factors: $3 / 2,2 / 3,5 / 3$ for $8,3^{*}$ and 6 , respectively, implying that the color-sextet channel may diverge most strongly.

\section{Numerical Results}

\subsection{Simulation parameters and statistics}

We perform $S U$ (3) lattice gauge simulations in the quenched approximation to calculate the color decomposed PPL correlators. The lattice update was done by the heat-bath Monte Carlo algorithm with a plaquette Wilson gauge action. The lattice configuration numbers for the $18^{4}, 24^{4}$ and $32^{4}$ lattices are 600, 700 and 320; additionally, in order to investigate the volume dependence, we added the $8^{4}$ and $12^{4}$ lattices with 200 configurations. The lattice configurations updated are fixed to the Coulomb gauge by the iterative gauge fixing method [22], and we also use the temporal gauge fixing that does not affect the Coulomb gauge feature. The lattice coupling constant $\beta$ for all the lattices is fixed to 5.9 corresponding to the lattice cut-off $a \sim 0.12 \mathrm{fm}$ [25].

\subsection{Color-dependent potentials}

Figure 1 shows numerical results for the color-Coulomb instantaneous potential $V(R, 0)$, in the color-singlet, color-octet, color-sextet, color-triplet (antisymmetric) channels. We find that both the color-singlet $V_{1}$ and color-antitriplet $V_{3^{*}}$ potentials yield attractions at all distances, and are linearly rising potential at large distances. On the other hand, the color-octet $V_{8}$ and color-sextet $V_{6}$ potentials are repulsive forces. 


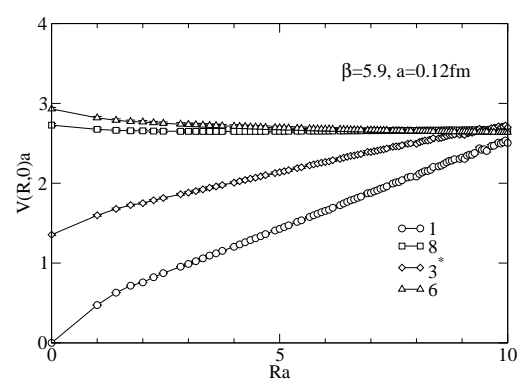

Figure 1: Lattice numerical results of color-Coulomb instantaneous potentials between two quarks. $(\beta=$ $5.9, a \sim 0.12 \mathrm{fm})$

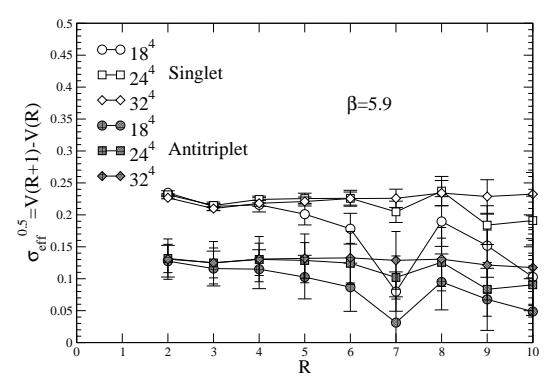

Figure 2: Effective string tensions for the colorsinglet and color-antitriplet channels in lattice units.

We calculate an effective string tension for the color-singlet and color-antitriplet channels. Here the effective string tension is defined as $K=V(R+1)-V(R)$ in lattice units, which should be a constant for large quark separations if it is a confining potential with a finite string tension. In Fig. 2 we find that the Ks become stable over approximately $R=3 \sim 4$ as the lattice size increases. When we use the data for $R=3-6\left(R=3-5\right.$ for the $18^{4}$ lattice), we plotted in Fig. 3 the ratio $K_{1} / K_{3^{*}}$, which is found to be close to $C_{1} / C_{3^{*}}=2$. Note that our definition of the color-Coulomb instantaneous part on a lattice in terms of the PPL correlator (Eq. (2.8)) does not completely exclude a vacuum polarization effect.

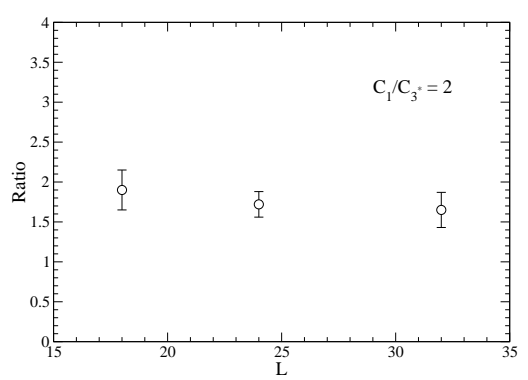

Figure 3: The vertical axis stands for the ratios $K_{1} / K_{3^{*}}$ of the string tensions in the singlet and antitriplet channels and the horizontal axis is the spatial lattice size.

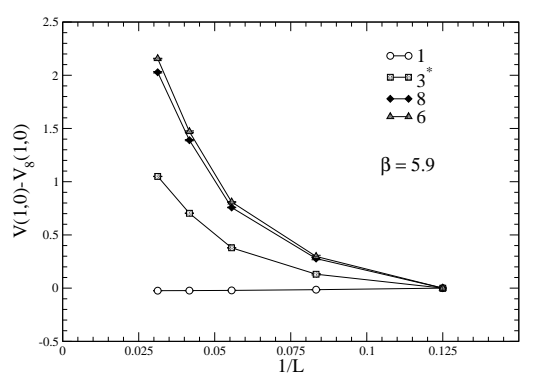

Figure 4: The volume dependence for the colordependent instantaneous potentials at the distance $R=$ 1 scaled by the $V_{8}(1,0)$ on the 8 cubic lattice.

\subsection{Divergence of color-nonsinglet potentials}

Here we consider the infrared divergence property of the color-dependent potentials while the string tensions obtained from them have little volume dependence as shown in the previous section. The short-distance Coulomb term $\sim 1 / R$, which is not related to the color confinement, may not matter on this argument. Figure 4 shows our non-perturbative numerical results, by which we find that the color-singlet potential has little volume dependence and the absolute value of the 
color-nonsinglet potentials increases with the lattice volume. In addition, Fig. 4 implies that the magnitude of the divergence on the finite lattice seems to depend on the ratio being $4: 9: 10$ for $3^{*}$, 8 and 6 as observed in the section II. E; however, note that they will diverge equally to infinity in the large volume limit. This indicates that the color-nonsinglet quarks can not exist independently; finally, they will become a color-singlet state with finite energy to compensate insufficient color degrees of freedom.

This numerical result does not contradict the Dual-Ginzbulg-Landau picture, in which one understands that the color-singlet flux between two quarks is shrunk like a string while the colornon-singlet flux will radiate rather than produce a closed string.

\section{Summary}

We have tried to calculate the long-distance color-dependent confining forces between two quarks in the quenched $S U(3)$ lattice simulation with the Polyakov line correlator. We here focus the color-Coulomb instantaneous term in the Coulomb gauge QCD, which has been discussed in the Gribov-Zwanziger confinement scenario and is required to make the hadron bound state consisting of quarks.

Our numerical simulation shows that the color-singlet $q \bar{q}$ channel as well as the color-antitriplet $q q$ (di-quark) channel causes a linearly confining potential at large distances. The other color-octet and color-sextet channels at large distances yield also repulsive forces which are weaker than the attractive ones. In addition, we find that the string tensions in the color-singlet and color-antitriplet channels have not significant volume dependence.

We also investigated the infrared divergence of color-non-singlet potentials and find that the divergence on the finite lattice seems to be proportional to the color (Casimir) factor. In the infinite volume limit the color-dependent potentials except the color-singlet channel will diverge. This conclusion is consistent with the Dual-Ginzburg-Landau picture of the color confinement.

This approach that we take notice of the color-Coulomb instantaneous part may be suitable for further work to investigate three-quark color-dependent forces for the understanding of baryons as well as new multiquark particles. It is also necessary to study how the vacuum polarization term affects the instantaneous forces because it is reported in Refs. [11,26] that the octet (adjoint) channel calculated by the Polyakov line correlator with the vacuum polarization gives the complicated distance dependence at large distances.

\section{Acknowledgments}

The simulation was performed on SX-5 and SX-8 (NEC) vector-parallel computers at the RCNP of Osaka University. We appreciate the warm hospitality and support of the RCNP administrators.

\section{References}

[1] D. Zwanziger, Nucl. Phys. B 518 (1998) 237-272.

[2] L. Baulieu, D. Zwanziger, Nucl. Phys. B548 (1999) 527-562. 
[3] A. Niégawa, Phys. Rev. D74, 045021 (2006); A. Niégawa, M. Inui, and H. Kohyama, Phys. Rev. D 74, 105016 (2006).

[4] J. Greensite and Š. Olejník, Phys. Rev. D67, 094503 (2003).

[5] J. Greensite, Š. Olejník and D. Zwanziger, Phys. Rev. D69, 074506(2004).

[6] A. Nakamura, T. Saito, Prog.Theor.Phys. 115 (2006) 189-200.

[7] Y. Nakagawa, A. Nakamura, T. Saito, H. Toki, D. Zwanziger, Phys.Rev. D73 (2006) 094504.

[8] J. Greensite, Š. Olejník and D. Zwanziger, JHEP 0505 (2005) 070.

[9] Y. Nakagawa, A. Nakamura, T. Saito, H. Toki, Phys. Rev. D75 (2007) 014508.

[10] V. N. Gribov, Nucl. Phys. B139, 1 (1978).

[11] A. Nakamura, T. Saito, Phys. Lett. B621(2005) 171-175.

[12] S. Nadkarni, Phys. Rev. D 33, 3738 (1986); Phys. Rev. D 34, 3904 (1986).

[13] M. Hess, F. Karsch, E. Laermann, and I. Wetzorke, Phys. Rev. D58, 11502.

[14] K. Orginos, PoS (LAT2005) 054.

[15] C. Alexandrou, P. de Forcrand, B. Lucini, PoS (LAT2005) 053.

[16] Z. Fodor, C. Hölbling, M. Mechtel, K. Szabo, PoS (LAT2005) 310.

[17] A. Nakamura and T. Saito, Prog.Theor.Phys. 112 (2004) 183-188.

[18] A. Nakamura and T. Saito, Prog.Theor.Phys. 111 (2004) 733-743.

[19] Y. Maezawa, N. Ukita, S. Aoki, S. Ejiri, T. Hatsuda, N. Ishii, and K. Kanaya, Phys. Rev. D 75 (2007) 074501 .

[20] M. Döring, K. Hübner, O. Kaczmarek, F. Karsch Phys. Rev. D75 (2007) 054504.

[21] D. Zwanziger, Phys. Rev. Lett. 90 (2003) 102001.

[22] J.E. Mandula and M. Ogilvie, Phys. Lett. B185 (1987) 127.

[23] D. Zwanziger, Nucl. Phys. B485 (1997) 185-240.

[24] P.O. Bowman and A.P. Szczepaniak, Phys. Rev. D 70, 016002 (2004).

[25] K. Akemi, et al., QCDTARO Collaboration, Phys. Rev. Lett. 71 (1993) 3063.

[26] O. Philipsen, Phys. Lett. B535 (2002) 138-144.

[27] K. Langfeld and L. Moyaerts, Phys. Rev. D70 (2004) 074507. 